\title{
PLAGUICIDAS ORGANOFOSFORADOS Y CÁNCER \\ EN LATINOAMÉRICA: \\ EVIDENCIA PARA UNA DISCUSIÓN BIOÉTICA
}

\section{ORGANOPHOSPHATE PESTICIDES AND CANCER IN LATIN AMERICA: EVIDENCE FOR A BIOETHICAL DISCUSSION}

\section{DRA. MARÍA TERESA MUÑOZ QUEZADA}

mtmunoz@ucm.cl

\section{DR. BORIS ANDRÉS LUCERO MONDACA}

Facultad de Ciencias de la Salud

Universidad Católica del Maule. Talca, Chile.

DRA. VERÓNICA PAZ IGLESIAS ÁLAMOS

MG. MARÍA PÍA MUÑOZ

DRA. CARMEN ANTINI IRRIBARRA

\section{MG. NATALIA LUCERO}

Facultad de Medicina

Universidad de Chile. Santiago, Chile.

DOI: http://doi.org/10.29035/ucmaule.53.93

\section{RESUMEN}

Existe evidencia de que la exposición a plaguicidas altamente peligrosos se asocia con el desarrollo de cáncer. Los plaguicidas organofosforados (OP) están dentro de este grupo y son muy utilizados en Latinoamérica. El objetivo fue analizar estudios latinoamericanos sobre la exposición a plaguicidas organofosforados y sus efectos en el desarrollo de cáncer humano y discutir sus implicancias bioéticas. Para ello, se realizó una búsqueda sistemática en PubMed, replicándola luego en otras bases de datos. Los criterios de elegibilidad fueron: estudios científicos latinoamericanos, muestras de población o tejido humano, exposición a OP y desarrollo de cáncer. Los términos de búsqueda fueron organofosforados, cáncer y el nombre de cada país. De un total de 1092 artículos, 11 cumplieron con los criterios. Todos encontraron relación entre exposición a plaguicidas OP y desarrollo de cáncer. El $81 \%$ de los estudios eran experimentales y demostraron que la exposición a clorpirifos, paratión o malatión provocan el desarrollo de carcinoma mamario humano. Se concluye que las investigaciones latinoamericanas apuntan 
a que la exposición a plaguicidas OP genera cáncer en humanos, por lo tanto se requiere el desarrollo de políticas que regulen su uso y prohibición de los más peligrosos.

Palabras clave: Cáncer, Latinoamérica, Organofosforados, Salud Pública, Bioética.

\section{ABSTRACT}

There is evidence that the exposure to highly hazardous pesticides is associated with cancer development. Organophosphate pesticides (OP) are within this group and are widely used in Latin America. The aim of this paper was to review the Latin American research on exposure to organophosphate pesticides and its effects on the development of human cancer and discuss their bioethical implications. A systematic search was run in PubMed database, then replicating it in other databases. Eligibility criteria were: Latin American scientific studies, samples of human population or tissue, OP exposure and cancer development. Search terms were organophosphates, cancer and the name of each country. Of a total of 1092 articles, 11 met the criteria. All found relationship between OP pesticide exposure and cancer development. $81 \%$ of the studies were experimental and showed that exposure to chlorpyrifos, parathion and malathion cause the development of human breast carcinoma. We conclude that Latin American research suggests that OP pesticide exposure generates cancer in humans; therefore, the development of policies that regulate the use of pesticides and forbid the most dangerous is required.

Key words: Cancer, Latin America, Organophosphates, Public Health, Bioethics. 


\section{INTRODUCCIÓN}

Los pesticidas organofosforados (OP) son sustancias químicas utilizadas para el control y exterminación de plagas, principalmente insectos, siendo tóxicos para los seres humanos y el medio ambiente (Levine, 2007; Thundiyil, Stober, Besbelli y Pronczuk, 2008). Sin embargo, su bajo costo explica su amplio uso a nivel mundial (Thundiyil et al., 2008). El fósforo liberado en un organismo intoxicado con OP se asocia a la acetilcolinesterasa, inhibiendo la metabolización de la acetilcolina. La acumulación de este neurotransmisor en las sinapsis colinérgicas provoca una sobreestimulación de los receptores muscarínicos y nicotínicos del sistema nervioso central, causando intoxicaciones agudas y crónicas (Barr et al., 2006; Costa, 2006). Los procesos de hidrólisis hepática de los OP, junto con el glutatión y las oxidasas, potencian la toxicidad de los metabolitos derivados. Las principales vías de exposición a OP son la ocupacional, el consumo de alimentos con residuos de OP, residir cerca de predios agrícolas y la exposición intradomiciliaria (Lu, Barr, Pearson y Waller, 2008; Muñoz-Quezada et al., 2012; Rodríguez et al., 2006; Vida y Moretto, 2007).

Diversos estudios internacionales realizados en trabajadores agrícolas y sus hijos, evidencian los efectos agudos -náuseas, problemas respiratorios, vómitos, bradicardia, miosis, dermatitis, quemaduras- y crónicos -deficiencias en las funciones cognitivas, motoras, sensoriales, enfermedades neurológicas- en el ser humano (Barr et al., 2006; Engel, Berkowitz, Barr, Teitelbaum, Siskind y Meisel, 2007; Eskenazi, Mars, Bradman, Harley, Barr, Johnson, Morga y Jewell, 2007). La intensidad del cuadro clínico puede variar desde leve hasta fatal (Alavanja, Hoppin y Kamel, 2004; Barr et al., 2006). Otros efectos relevantes son retardo del crecimiento intrauterino, trastornos neuroconductuales y alteraciones endocrinas e inmunotóxicas (Eskenazi et al., 2004; Eskenazi et al., 2007; Jurewicz y Hanke, 2008; Needham, 2005; Rosas y Eskenazi, 2008).

Reportes provenientes en su mayoría de Estados Unidos y Canadá evidencian específicamente que la exposición ocupacional e intradomiciliaria a OP produciría cáncer, leucemia, linfomas y tumores (Alavanja et al., 2003; Bonner, Coble y Blair, 2007; Jurewicz y Hanke, 2008; Lee et al., 2004; Meinert, Schuz, Kaletsch, Kaatsch y Michaelis, 2000; Waddell et al., 2001). Sin embargo, estos estudios aún plantean dificultades en la evaluación de la exposición.

A diferencia de Latinoamérica, en Estados Unidos, Canadá y Europa (MuñozQuezada, 2011) está prohibido el uso de OP tales como diazinón, dimetoato, 
malatión y metamidofos, además existe una estricta regulación y control de otros pesticidas como el clorpirifos. Lo anterior, unido a la ausencia de información sistematizada sobre la asociación entre exposición a OP y al desarrollo de cáncer en seres humanos, y al hecho de que esta patología es la que genera uno de los mayores daños a nivel individual y social, con una letalidad a corto plazo, indica la conveniencia de revisar la literatura latinoamericana existente en este tema y discutir sus implicancias bioéticas para la región.

\section{MÉTODO}

Para analizar los datos disponibles, se identificaron estudios en todos los países de la región hasta el año 2014. Se utilizó la técnica de la búsqueda sistemática, la cual se inició con el buscador PubMed y se replicó en EBSCOhost, LILACS y SciELO con el fin de contrastar los resultados obtenidos y agregar artículos ausentes en PubMed.

Los términos de búsqueda fueron "Organofosforados" y el nombre de 23 países latinoamericanos, cada uno por separado, ingresados en inglés y en español. Posteriormente, se buscaron las palabras "Malatión", "Paratión" y "Clorpirifos", y "Organofosforados" con "Cáncer" para cada país. Se revisó para cada estudio encontrado la muestra, diseño, medición de exposición, medición de efectos y resultados. Los criterios de elegibilidad fueron: estudios empíricos realizados en Latinoamérica, muestras de población o tejido humano, exposición a OP y cáncer.

Los artículos seleccionados fueron evaluados utilizando un criterio descrito en revisiones sistemáticas realizadas a nivel internacional (Muñoz-Quezada, 2013; Rohlman, Anger y Lein, 2011), según el cual se asigna una calificación en base a: (1) diseño del estudio, (2) tamaño de la muestra, (3) evaluación de la exposición, (4) evaluación del efecto y (5) control de confusores, asignando una puntuación de 0 a 2 para cada uno de los 5 parámetros mencionados (Tabla 1). Finalmente, se clasifican los estudios en tres categorías: baja (0-2 puntos), intermedia (3-7 puntos) y alta (8-10 puntos).

La búsqueda, puntuación y clasificación de los artículos fue realizada por cuatro investigadores por separado. En caso de existir controversias, estas fueron resueltas por un quinto investigador. 
Tabla 1

Definición de los criterios para el puntaje de calificación para evaluar el estándar de calidad de los estudios incluidos en la revisión.

\begin{tabular}{|c|c|c|c|}
\hline Parámetros & 2 & 1 & 0 \\
\hline $\begin{array}{l}\text { 1. Diseño del } \\
\text { estudio }\end{array}$ & $\begin{array}{l}\text { Longitudinal, } \\
\text { exposición anterior } \\
\text { al outcome, } \\
\text { experimental. }\end{array}$ & $\begin{array}{l}\text { Caso control, } \\
\text { transversal } \\
\text { analítico. }\end{array}$ & $\begin{array}{l}\text { Estudio de } \\
\text { caso, ecológico, } \\
\text { exploratorio, } \\
\text { descriptivo. }\end{array}$ \\
\hline $\begin{array}{l}\text { 2. Tamaño de la } \\
\text { muestra }\end{array}$ & $>200$ & Entre 50 y 200 & $<50$ \\
\hline $\begin{array}{l}\text { 3. Evaluación de la } \\
\text { exposición }\end{array}$ & $\begin{array}{l}\text { Biomarcadores en } \\
\text { orina. } \\
\text { Tratamiento directo } \\
\text { con pesticidas } \\
\text { OP en las células } \\
\text { (experimental). }\end{array}$ & $\begin{array}{l}\text { Biomarcadores } \\
\text { generales (ej. } \\
\text { acetilcolinesterasa). }\end{array}$ & $\begin{array}{l}\text { No especifica } \\
\text { biomarcadores } \\
\text { de exposición } \\
\text { a OP (Estudio } \\
\text { ecológico, registros } \\
\text { de hospitales, } \\
\text { cercanía a predios, } \\
\text { ocupación, etc.). }\end{array}$ \\
\hline $\begin{array}{l}\text { 4. Evaluación del } \\
\text { efecto (cáncer) }\end{array}$ & $\begin{array}{l}\text { Uso de pruebas } \\
\text { de laboratorio } \\
\text { estandarizadas } \\
\text { y pruebas } \\
\text { específicas. }\end{array}$ & $\begin{array}{l}\text { Test de screening } \\
\text { e instrumentos } \\
\text { estandarizados no } \\
\text { validados. }\end{array}$ & $\begin{array}{l}\text { Uso de } \\
\text { cuestionarios, } \\
\text { entrevistas y fichas } \\
\text { clínicas. }\end{array}$ \\
\hline $\begin{array}{l}\text { 5. Control de } \\
\text { confusores }\end{array}$ & $\begin{array}{l}\text { Control de } \\
\text { confusores } \\
\text { importantes } \\
\text { (estudio de } \\
\text { laboratorio, } \\
\text { enfermedad, } \\
\text { exposición a otros } \\
\text { tóxicos) y Variables } \\
\text { estándar. }\end{array}$ & $\begin{array}{l}\text { Variables estándar } \\
\text { controladas en } \\
\text { análisis (ej: edad, } \\
\text { sexo, ocupación). }\end{array}$ & $\begin{array}{l}\text { No considera } \\
\text { variables estándar } \\
\text { y confusores o no } \\
\text { se realiza un buen } \\
\text { análisis estadístico } \\
\text { de confusores. }\end{array}$ \\
\hline
\end{tabular}




\section{RESULTADOS}

La búsqueda avanzada con la palabra clave "Organophosphate" y el nombre de cada país latinoamericano en PubMed identificó un total de 1092 artículos en idioma inglés. Al revisar los resúmenes, se constató que solo 10 cumplían con los criterios de elegibilidad. De estos, ocho eran chilenos, uno de Argentina y uno de Costa Rica. Se replicó la búsqueda utilizando las otras palabras clave, sin identificar más artículos. En el buscador SciELO se identificó un artículo bajo las palabras "Malatión" y "Cáncer", realizado en Chile. Los buscadores EBSCOhost y LILACS no entregaron más artículos. El total de artículos que cumplió con los criterios de elegibilidad establecidos fue 11.

La Tabla 2 presenta una síntesis del objetivo, diseño, muestra, medición de la exposición y del efecto, evaluación de la calidad y resultados. En ella se observa que todos los estudios analizados encontraron una relación dosis-respuesta a OP. De estos, nueve clasificaron en un nivel de calidad alto, correspondiendo a investigaciones experimentales sobre exposición controlada a malatión, paratión y clorpirifos y desarrollo de cáncer mamario en tejido humano, realizadas en Chile y Argentina (Cabello, Juarranz, Botella y Calaf, 2003; Cabello et al., 2003; Calaf y Roy, 2007a; Calaf y Roy, 2007b; Calaf y Roy, 2007c; Calaf y Roy, 2008a; Calaf y Roy, 2008b; Calaf, Echiburu-Chay y Roy, 2009; Ventura et al., 2012).

De acuerdo con los resultados obtenidos por el equipo de Cabello (2003), se evidenció que el paratión y el malatión aumentaron el antígeno nuclear de células en proliferación (PCNA), induciendo la expresión de la proteína mutante p53 de las líneas celulares de cáncer de mama estrógeno-dependiente (MCF7), en comparación a los controles y los tejidos tratados con atropina, en los cuales se inhibió tal acción. Esto indicaría que los OP podrían inducir más cambios en esta línea celular maligna de la mama. 


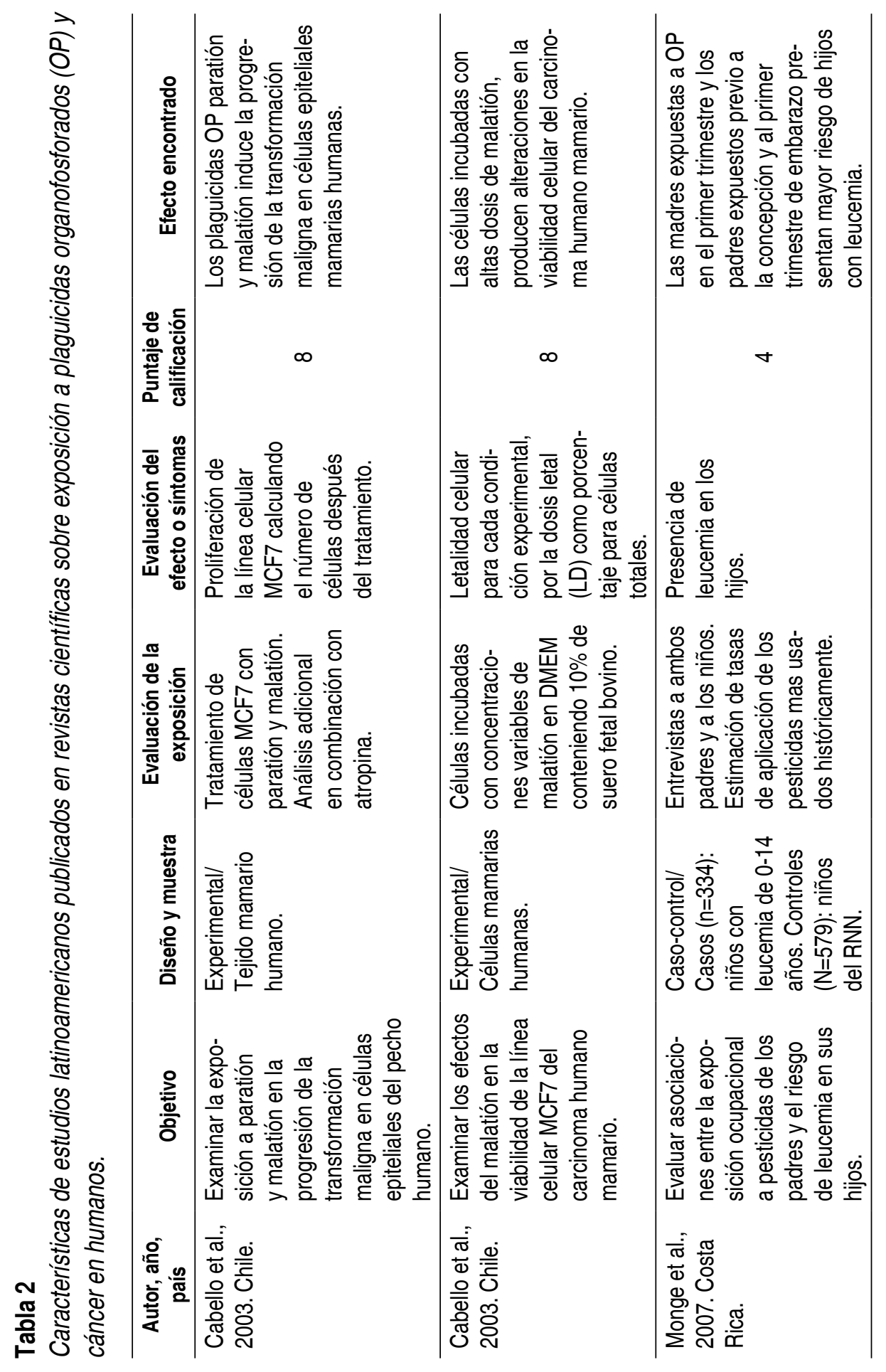




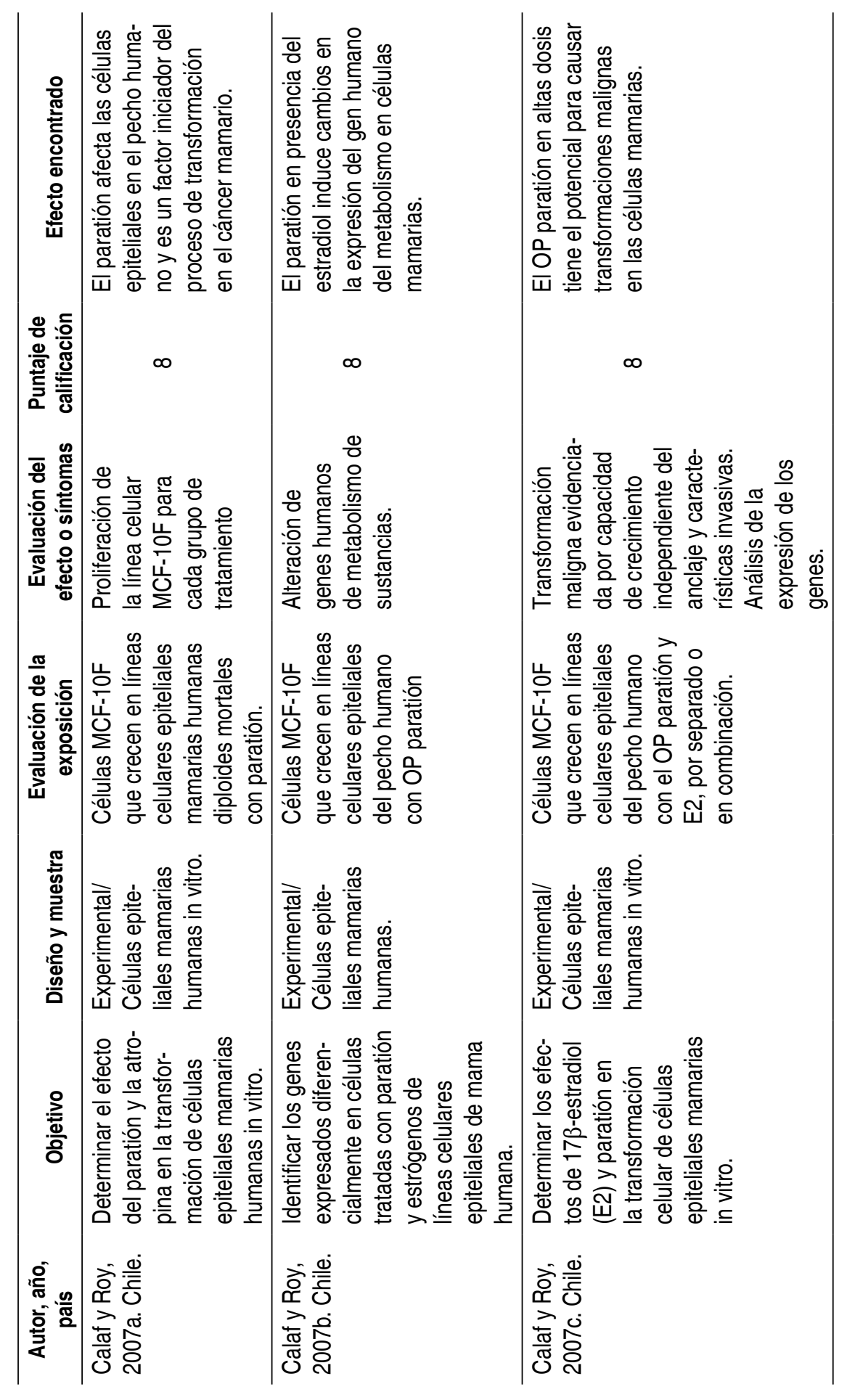




\begin{tabular}{|c|c|c|}
\hline 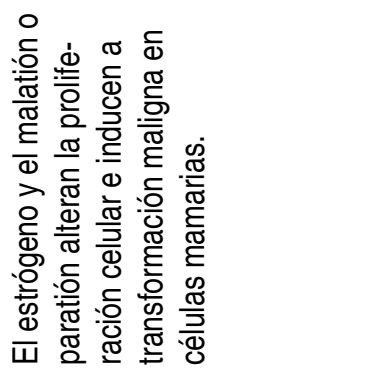 & 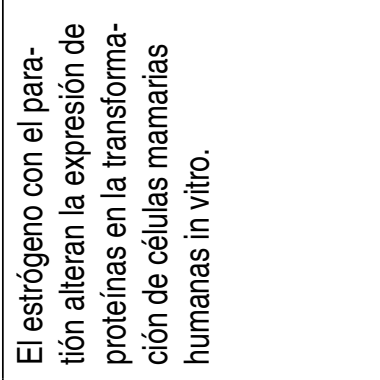 & 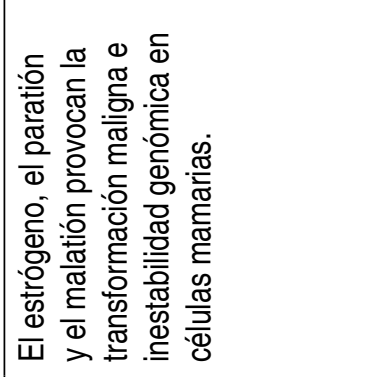 \\
\hline$\infty$ & $\infty$ & $\infty$ \\
\hline 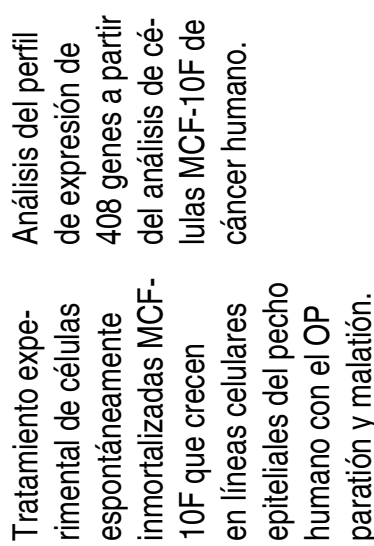 & 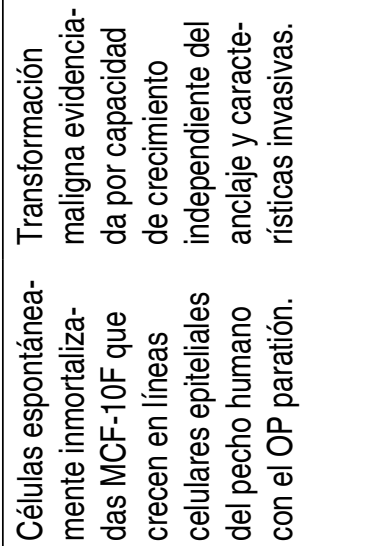 & 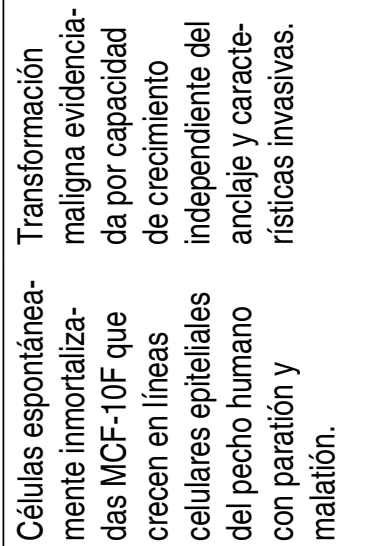 \\
\hline 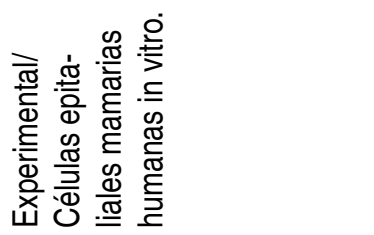 & 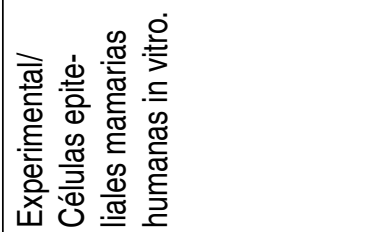 & 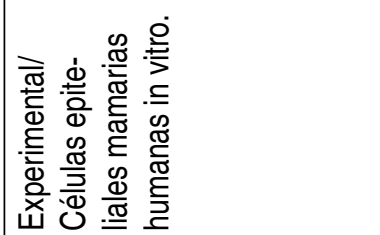 \\
\hline 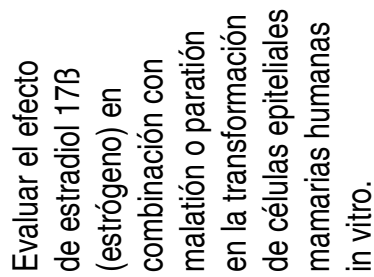 & 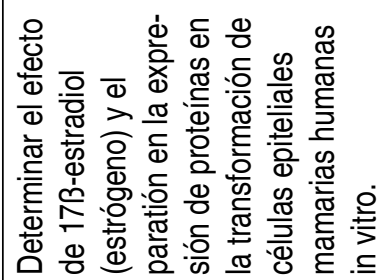 & 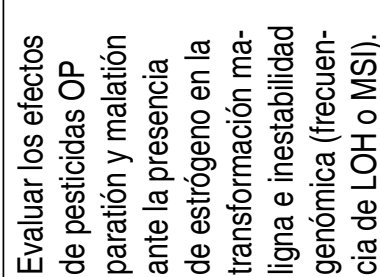 \\
\hline 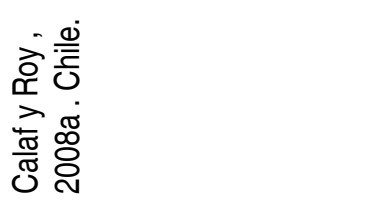 & 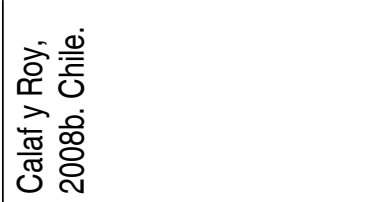 & 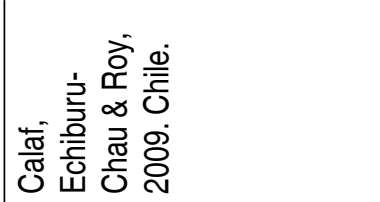 \\
\hline
\end{tabular}




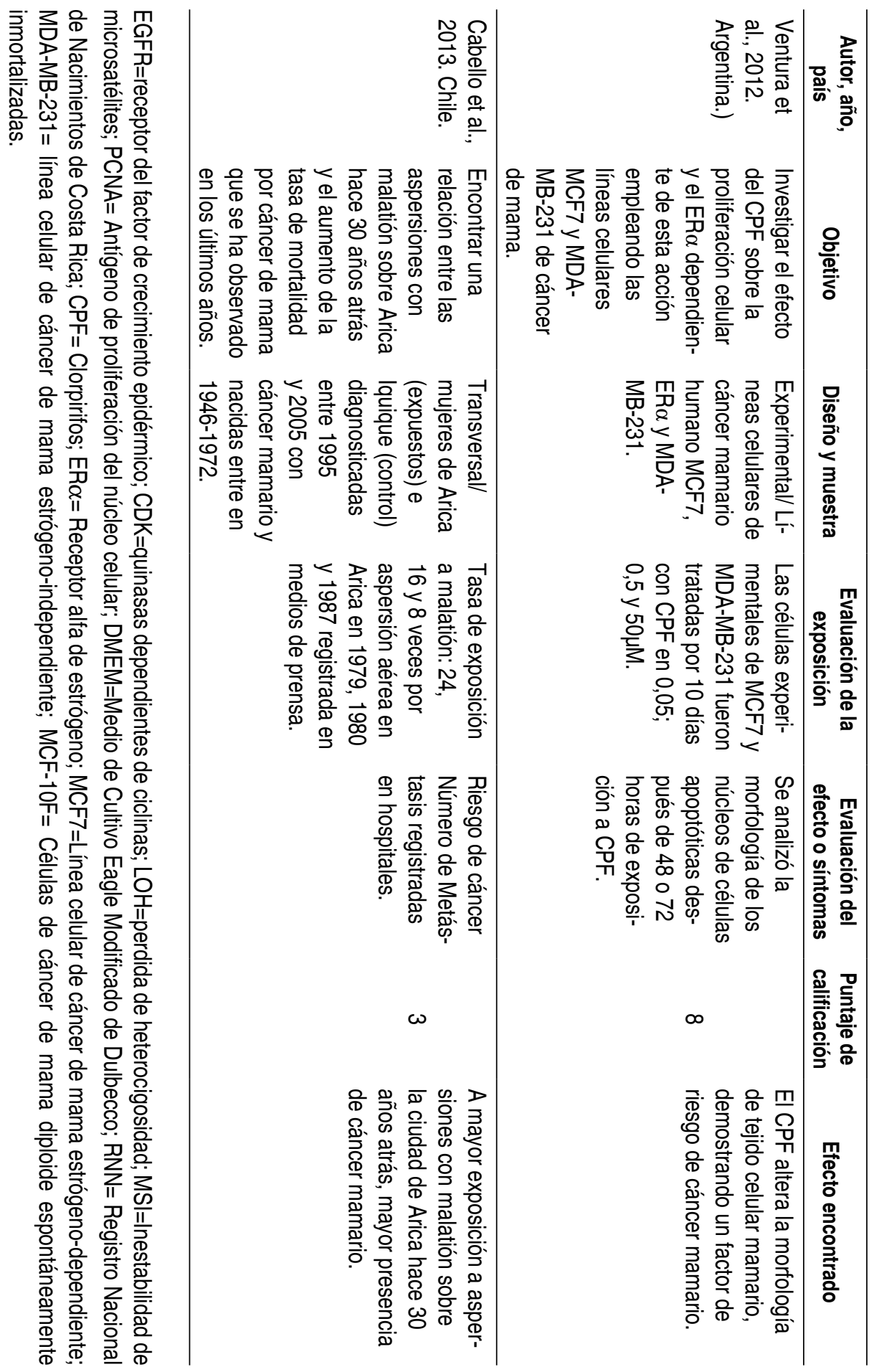


Un segundo estudio experimental, publicado por Cabello et al. (2003), examina los efectos del malatión en la viabilidad de las células MCF7 del carcinoma humano mamario, evaluando también las alteraciones provocadas por este pesticida en la adhesión celular y su relación con E-cadherina, $\beta$-catenina y los microfilamentos de actina. Este grupo reporta que las células incubadas con bajas concentraciones de malatión (16-32 $\mu \mathrm{g} / \mathrm{ml})$ mostraron altos niveles de supervivencia (> 95\%) en todos los tiempos evaluados ( 1 a 5 días), en contraste, al usar $512 \mu \mathrm{g} / \mathrm{ml} \mathrm{de}$ malatión y cinco días de tratamiento, la letalidad fue total. El uso de altas dosis de este pesticida (64 y $128 \mu \mathrm{g} / \mathrm{ml}$ ) durante cinco días de tratamiento indujo cambios morfológicos profundos (vacuolas perinucleares, redondeo, contracción y pérdida gradual de adhesión) en las células analizadas. Estos se asociaron a la disminución en la expresión de las moléculas de adhesión E-cadherina y $\beta$-catenina y de la distribución y reactividad de los microfilamentos de actina al Tetramethylrhodamina Isothiocynada (TRITC-phalloidin). La disrupción de los microfilamentos, acompañados por el colapso de la actina en las regiones perinucleares, fueron características en células con pérdida de adhesión. A menores concentraciones de malatión, algunas células presentaron deformaciones en la membrana plasmática en estructura tipo-lamellipodia, particularmente evidentes desde los 32 a los 128 $\mu \mathrm{g} / \mathrm{ml}$.

Calaf y Roy (2007a) realizaron tres publicaciones sobre los efectos del paratión sobre células epiteliales mamarias humanas. En el primero de ellos, se observó que el paratión por si solo indujo transformaciones malignas en las líneas celulares MCF-10F. Las células tratadas con paratión mostraron un incremento de c-kit, Trio, Rho-A, Rac-3, EGFR, Notch-4, Dvl-2, Ezrin, $\beta$ catenina y proteína p53 mutante, sin embargo la atropina inhibió significativamente este crecimiento. En una muestra de ciclo celular humano de 96 genes, 13 fueron alteradas por el paratión. Los autores concluyeron que el paratión influye en la transformación de células epiteliales en el pecho humano y es un factor iniciador del proceso de transformación maligna del cáncer mamario.

El segundo reporte (Calaf y Roy, 2007b), evidencia alteración en 17 genes, ya sea por paratión o estrógenos por separado o por la combinación de ambos, concluyendo que, en presencia de estradiol, pesticidas OP como el paratión inducen cambios en la expresión del gen humano del metabolismo en las células mamarias. Una tercera publicación (Calaf y Roy, 2007c), analiza los efectos de 17ß-estradiol (E2) y el paratión en la transformación de células epiteliales mamarias in vitro, informando que este pesticida por si solo o en combinación con E2 induce transformación maligna en células MCF-10F, la que es confirmada 
por independencia del anclaje y por sus capacidades invasivas. Además, se observaron alteraciones transcripcionales en 22 de 96 genes, entre ellos, nueve involucrados en el ciclo celular, por lo que concluyeron que el paratión provoca transformación maligna de las células mamarias epiteliales a través de la modulación de la expresión de genes regulados por el ciclo celular.

Calaf y Roy (2008a) informaron acerca de la transformación de células epiteliales mamarias humanas in vitro en relación a la combinación de estradiol $17 \beta$ con malatión o paratión. Los ensayos mostraron que el E2 en combinación ya sea con malatión o paratión, alteraba la proliferación celular e inducía transformación maligna, exhibiendo capacidades invasivas significativas al compararlas con las células de control de la línea MCF-10F. A partir de estos resultados, los autores señalaron que los pesticidas y los estrógenos afectan las células mamarias humanas induciendo cambios moleculares indicativos de transformación.

Un segundo estudio de Calaf y Roy (2008b), que analiza el efecto del $17 \beta$-estradiol y del paratión en la expresión de proteínas relacionadas con la transformación de células epiteliales mamarias, informa que E2 y paratión, tanto por separado como combinados, inducen una transformación maligna en células MCF-10F, lo que es confirmado por independencia del anclaje y sus capacidades invasivas. La combinación de E2 y paratión aumentó la expresión de proteínas asociadas a la adhesión celular. Los autores concluyeron que este pesticida produce cambios en la adhesión de las células mamarias humanas, que constituyen indicativos de transformación maligna.

En otro estudio, Calaf et al. (2009) evaluaron los efectos de pesticidas OP paratión y malatión ante la presencia de E2 en la transformación maligna e inestabilidad genómica e informaron que estos pesticidas, por separado 0 en combinación con E2 inducen transformación maligna en células mamarias, evidenciado por un incremento significativo en la expresión de las proteínas p53 y c-Ha-ras, cruciales para el proceso del cáncer.

Cabello et al. (2013) evaluaron la relación entre las 24 aspersiones con malatión realizadas sobre la ciudad de Arica en 1983 y el aumento en la tasa de mortalidad por cáncer de mama observada en dicha ciudad en los últimos años. Los autores encontraron que las mujeres con mayor tiempo de exposición a malatión fueron 5.7 veces más propensas a ser diagnosticadas con cáncer mamario $(\mathrm{OR}=5.7$, $p<0.02$ ), evidenciándose además metástasis en un $30.5 \%$ del grupo expuesto y un $16 \%$ del grupo no expuesto. Estos hallazgos permitieron concluir que el 
aumento en la mortalidad por cáncer mamario en Arica se asocia de manera significativa con la exposición al malatión pulverizado sobre la ciudad en la década de los ochenta. Sin embargo, para obtener los resultados de este estudio no se entregaron antecedentes asociados a los diagnósticos de las mujeres y no se evaluó la exposición con biomarcadores.

Siguiendo una línea similar a los estudios anteriores, en Argentina se demostró (Ventura et al., 2012), que la exposición a $0.05 \mu \mathrm{M}$ de clorpirifos (CPF) induce la proliferación celular a través del receptor alfa de estrógeno $(E R \alpha)$ en células

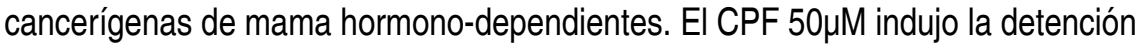
de la fase intra-S (fase encargada de regular la replicación en cromosomas humanos) modificando puntos de control de proteínas, a través de un mecanismo que puede implicar cambios en el equilibrio de las células MCF7, mientras que en líneas celulares estrógeno-independiente (MDA-MB 231) detiene la fase G2/M relacionada con la capacidad del plaguicida para unirse a sitios de tubulina, alterando la polimeración de los microtúbulos. Este estudio entrega evidencia novedosa de que el CPF posee un factor de riesgo ambiental de cáncer mamario.

Finalmente, el estudio de caso y control realizado por Monge y colaboradores en Costa Rica (Monge et al., 2007), reporta un riesgo aumentado de leucemia en niños asociado a la exposición materna y paterna a OP durante el primer trimestre del embarazo $(\mathrm{OR}=3.5 ; \mathrm{IC} 95 \%=1.0-12.2$ y OR = 1.6; IC95\% = $1.0-2.6$, respectivamente) y a la exposición paterna en el año previo a la concepción ( $\mathrm{OR}=$ 1.5; IC95\% = 1.0 - 2.2). Sin embargo, esta investigación determinó la exposición a partir de un modelo construido desde fuentes secundarias con datos de diverso origen y por medio de entrevistas con los mismos expuestos, aumentando en este último caso la posibilidad de sesgo de memoria en los participantes.

\section{Discusión}

Los estudios realizados en Latinoamérica demuestran que la exposición a plaguicidas OP se asocia con cáncer. De los 11 artículos que cumplieron con los criterios de elegibilidad, nueve midieron exposición a plaguicidas OP a través de células humanas tratadas directamente con OP y son de alta calidad en el desarrollo de la investigación y en el control de sesgos (Cabello et al., 2003; Calaf y Roy, 2007a; Calaf y Roy, 2007b; Calaf y Roy, 2007c; Calaf y Roy, 2008a; Calaf y Roy, 2008b; Calaf et al., 2009; Ventura et al., 2012). Dentro de estas 
investigaciones, la presencia de OP en tejido celular humano o en el organismo se asocia a la proliferación y transformación maligna de células mamarias.

Los plaguicidas OP considerados en los estudios analizados fueron el paratión, malatión y CPF. Mediante la técnica de la entrevista también se evaluó la exposición a metamidofos y diclorvos y su relación con leucemia en hijos de trabajadores agrícolas. Esta información conlleva a una serie de implicancias bioéticas, las cuales se relacionan fundamentalmente con la necesidad de aplicar el principio precautorio. Este, de acuerdo a convenciones internacionales tales como la Declaración de Rio de Janeiro, aprobada por la Conferencia de las Naciones Unidas sobre el Medio Ambiente y el Desarrollo (Cafferatta, 2004), orienta a los estados a aplicar el criterio de precaución, desarrollando medidas eficaces ante el peligro de daño grave o irreversible, aún cuando no exista certeza científica absoluta. Esto implica adoptar un enfoque de prudencia y vigilancia con respecto a conductas y actividades efectiva o potencialmente lesivas.

En primer lugar, los plaguicidas mencionados corresponden a los más peligrosos según la OMS (Thundiyil et al., 2008; Red de Acción en Plaguicidas del Reino Unido, 2009). El insecticida paratión está prohibido en prácticamente todos los países latinoamericanos, sin embargo, el malatión, CPF y metamidofos aún se utilizan y se venden sin restricciones. Estudios latinoamericanos han encontrado residuos de estos plaguicidas en vegetales que consume la población general y metabolitos OP en la orina de escolares (Costa, 2006; Barr et al., 2006; Muñoz-Quezada et al., 2014). Además, como se mencionó en la introducción, la exposición a OP ocurre a través de la aplicación en el trabajo o en el hogar y por residir cerca de predios agrícolas (Eskenazi et al., 2007; Lu et al., 2008; MuñozQuezada et al., 2012; Rodríguez et al., 2006; Rothlein et al., 2006; Vida y Moretto, 2007). Por lo tanto, la población está expuesta por diferentes fuentes y vías que derivan en una serie de problemas de salud, en los que el cáncer se considera una de las enfermedades más graves por su rapidez, letalidad y deterioro de la calidad de vida de quienes lo vivencian y de sus familias.

Los estudios, desarrollados en laboratorios de alta complejidad, se concentran principalmente en el norte de Chile. Sería conveniente que en otros países de América Latina se fomente el desarrollo de evaluaciones de exposición a OP para identificar si el patrón varía entre zonas geográficas y en diferentes estaciones del año. Medir la exposición a OP en la población general, a través de biomarcadores como los metabolitos OP en orina, permitiría evaluar tendencias temporales y su comportamiento en respuesta a intervenciones para disminuir factores de 
riesgo. Estrategias similares han sido utilizadas en Estados Unidos, Canadá y en países europeos (Center for Disease Control and Prevention, 2009; European Commission, 2012; Health Canada, 2012).

En la población trabajadora, se debiera prestar atención a estudios observacionales de diseño longitudinal y transversal analítico que permitan evaluar el efecto de la exposición crónica a OP en trabajadores agrícolas, principalmente aplicadores y temporeras. Además, se requieren estudios epidemiológicos que evalúen la situación de salud de los trabajadores expuestos a pesticidas peligrosos que están prohibidos o limitados en su uso en Europa, Canadá y Estados Unidos, pero que en Latinoamérica aún se aplican (Red de Acción en Plaguicidas del Reino Unido, 2009). Actualmente, se desconoce la magnitud del problema en dicha población, tanto a nivel de exposición a estos pesticidas, como de sus efectos en la salud.

Una propuesta bioética para la región sería desarrollar medidas de comunicación y prevención del riesgo de exposición a residuos de plaguicidas. Se requiere promover medidas de información y educación a la población, relacionadas con la adecuada manipulación de frutas y vegetales, que probablemente presenten residuos de plaguicidas, y del riesgo en el uso de pesticidas en el hogar sin las debidas medidas de precaución. Al considerar la experiencia internacional en la materia, se sugiere diseñar regulaciones más específicas y estrictas sobre la presencia de residuos de plaguicidas en los alimentos, y generar una unidad de evaluación y medición del riesgo en la comida que se vende a la comunidad de forma periódica; además, es necesario crear modalidades de comunicación transparente decretadas por ley con respecto al tratamiento de los vegetales, cuidando el que resulten comprensibles para la población. Esto quiere decir, que se requiere acompañar con leyes estrictas de seguridad alimentaria las situaciones donde sea difícil alcanzar la certeza de ausencia de riesgo (Cafferatta, 2004; Van Dijk et al., 2008).

Finalmente, la producción y consumo de vegetales orgánicos es una alternativa que debe ser promovida y difundida en Latinoamérica. Algunos países ya cuentan con una normativa legal para la certificación de producción, procesamiento, almacenaje, transporte y comercialización de productos orgánicos agrícolas (Muñoz-Quezada et al., 2014), que resguarda la salud de los consumidores y el cuidado del medio ambiente. Por ende, se considera necesario a futuro evaluar si esta alternativa de producción disminuiría la exposición a pesticidas OP y sus posibles riesgos a la salud de la población. 


\section{Agradecimientos}

Este trabajo se desarrolló gracias al apoyo de la Facultad de Ciencias de la Salud de la Universidad Católica del Maule en el marco del proyecto PMI del Cáncer y los proyectos de la Comisión Nacional de Investigación Científica y Tecnológica CONICYT-FONIS SA13I20019 y FONDECYT 11150784.

\section{REFERENCIAS BIBLIOGRÁFICAS}

ALAVANJA, M., HOPPIN, J. Y KAMEL, F. (2004). Health effects of chronic pesticide exposure: cancer and neurotoxicity. Annu Rev of Public Health, 25, 155-197

ALAVANJA, M. C., SAMANIC, C., DOSEMECI, M., LUBIN, J., TARONE, R., LYNCH, C. F., et al. (2003). Use of agricultural pesticides and prostate cancer risk in the Agricultural Health Study cohort. Am J Epidemiol, 157(9), 800-814.

BARR, D. B., BRADMAN, A., FREEMAN, N., WHYATT, R. M., WANG, R. Y., NAEHER, L., et al. (2006). Studying the relation between pesticide exposure and human development. In: Belliger, D. C., editor. Human developmental neurotoxicology. Nueva York: Taylor \& Francis Group, p. 253-285.

BONNER, M. R., COBLE, J., BLAIR, A., BEANE FREEMAN, L. E., HOPPIN, J. A., SANDLER, D. P., et al. (2007). Malathion exposure and the incidence of cancer in the agricultural health study. Am J Epidemiol, 166(9), 1023-1034.

CABELLO, G., GALAZ, S., BOTELLA, L., CALAF, G., PACHECO, M., STOCKERT, J. C., et al. (2003). The pesticide malathion induces alterations in actin cytoskeleton and in cell adhesion of cultured breast carcinoma cells. A Int J Oncol, 23(3), 697704.

CABELLO, G., JUARRANZ, A., BOTELLA, L. M. Y CALAF, G. M. (2003). Organophosphorous pesticides in breast cancer progression. J Submicrosc Cytol Pathol, 35(1), 1-9. 
CABELLO, G., VALENZUELA-ESTRADA, M., SIQUES, P., BRITO, J., PARRA, E., VALDIVIA, $U$, et al. (2013). Relation of breast cancer and malathion aerial spraying in Arica, Chile. Int J Morphol, 31(2), 640-645.

CAFFERATTA, N. (2004). El principio precautorio. Gaceta Ecológica, 73, 5-21.

CALAF, G. M. Y ROY, D. (2008b). Cell adhesion proteins altered by $17 \square$ estradiol and parathion in breast epithelial cells. Oncol Rep, 19(1), 165-169.

CALAF, G. M. Y ROY, D. (2007a). Gene expression signature of parathiontransformed human breast epithelial cells. Int J Mol Med, 19(5), 741-750.

CALAF, G. M. Y ROY, D. (2007b). Human drug metabolism genes in parathionand estrogen-treated breast cells. Int J Mol Med, 20(6), 875-881.

CALAF, G. M. Y ROY, D. (2007c). Gene and Protein Expressions Induced by 17ß-estradiol and Parathion in Cultured Breast Epithelial Cells. Mol Med, 13(5-6), 255-265.

CALAF, G. M. Y ROY, D. (2008a). Cancer genes induced by malathion and parathion in the presence of estrogen in breast cells. Int J Mol Med, 21(2), 261-268.

CALAF, G. M., ECHIBURU-CHAU, C. Y ROY, D. (2009). Organophosphorous pesticides and estrogen induce transformation of breast cells affecting p53 and c-Ha-ras genes. Int J Oncol, 35(5), 1061-1068.

CENTERS FOR DISEASE CONTROL AND PREVENTION (2009). Fourth national report on human exposure to environmental chemicals. Recuperado de: http:// www.cdc.gov/exposurereport/pdf/FourthReport.pdf.

COSTA, L. G. (2006). Current issues in organophosphate toxicology. Clin Chim Acta, 366(1-2), 1-13.

ENGEL, S., BERKOWITZ, G., BARR, D., TEITELBAUM, S., SISKIND, J. Y MEISEL, S. (2007). Prenatal organophosphate metabolite and organochlorine levels and performance on the Brazelton Neonatal Behavioral Assessment Scale in a multiethnic pregnancy cohort. Am J Epidemiol, 265(12), 1397-1404. 
ESKENAZI, B., HARLEY, K., BRADMAN, A., WELTZIEN, E., JEWELL, N. P., BARR, D. B., et al. (2004). Association of in utero organophosphate pesticide exposure and fetal growth and length of gestation in an agricultural population. Environ Health Perspect, 112(10), 1116-1124.

ESKENAZI, B., MARKS, A. R., BRADMAN, A., HARLEY, K., BARR, D. B., JOHNSON, C., et al. (2007). Organophosphate pesticide exposure and neurodevelopment in young Mexican-American children. Environ Health Perspect, 115(5), 792-798.

EUROPEAN COMMISSION (2012). Health and consumers. Pesticides-EU rules. Recuperado de: http://ec.europa.eu/food/plant/pesticides/index_en.htm

GARRY, V. F. (2004). Pesticides and children. Toxicol Appl Pharmacol, 198(2), 152-163.

HEALTH CANADA (2012). Pesticides and pest management. Recuperado de: http://www.hc-sc.gc.ca/cps-spc/pest/index-eng.php

JUREWICZ, J. Y HANKE, W. (2008). Prenatal and childhood exposure to pesticides and neurobehavioral development: Review of epidemiological studies. Int J Occup Med Environ Health, 21(2), 121-132.

JUREWICZ, J. Y HANKE, W. (2008). Exposure to pesticides and childhood cancer risk: has there been any progress in epidemiological studies? Int $\mathrm{J}$ Occup Med Environ Health, 19(3), 152-169.

LEE, W. J., BLAIR, A., HOPPIN, J. A., LUBIN, J. H., RUSIECKI, J. A., SANDLER, D. P., et al. (2004). Cancer incidence among pesticide applicators exposed to chlorpyrifos in the Agricultural Health Study. J Natl Cancer Inst, 96(23), 1781-1789.

LEVINE, M. (2007). Pesticides: a toxic time bomb in our midst. USA: Praeger.

LU, C., BARR, D., PEARSON, M. Y WALLER, L. (2008). Dietary intake and its contribution to longitudinal organophosphorus pesticide exposure in urban/ suburban children. Environ Health Perspect, 116(4), 537-542. 
MEINERT, R., SCHUZ, J., KALETSCH, U., KAATSCH, P. Y MICHAELIS, J. (2000). Leukemia and non-Hodgkin's lymphoma in childhood and exposure to pesticides: results of a register-based case-control study in Germany. Am J Epidemiol, 151(7), 639-646.

MONGE, P., WESSELING, C., GUARDADO, J., LUNDBERG, I., AHLBOM, A., CANTOR, K. P., WEIDERPASS, E. Y PARTANEM, T. (2007). Parental occupational exposure to pesticides and the risk of chilhood leukemia in Costa Rica. Scand $J$ Work Environ Health, 33(4), 293-303.

MUÑOZ-QUEZADA M. T., LUCERO B., IGLESIAS, V., Y MUÑOZ M. P. (2014). Vías de exposición a plaguicidas en escolares de la Provincia de Talca, Chile. Gac Sanit 2014; 28(3): 190-195.

MUÑOZ-QUEZADA, M. T. (2011). Aspectos bioéticos en el control y aplicación de plaguicidas en Chile. Acta Bioeth, 17(1), 95-104.

MUÑOZ-QUEZADA, M. T., IGLESIAS, V., LUCERO, B., STEENLAND, K., BARR, D. B., LEVY, K., et al. (2012). Predictor of exposure to organophosphate pesticides in schoolchildren in the Province of Talca, Chile. Environ Int, 47, 28-36.

MUÑOZ-QUEZADA, M. T., LUCERO, B. A., BARR, D. B., STEENLAND, K., LEVY, K., RYAN, P. B., et al. (2013). Neurodevelopmental effects in children associated with exposure to organophosphate pesticides: A systematic review. Neurotoxicology, 39, 158-168.

NEEDHAM, L. L. (2005). Assessing exposure to organophosphorus pesticides by biomonitoring in epidemiologic studies of birth outcomes. Environ Health Perspect, 113(4), 494-498.

RED DE ACCIÓN EN PLAGUICIDAS DEL REINO UNIDO (2009). La lista de listas. Catálogo de listas de plaguicidas que identifican aquellos asociados con impactos particularmente dañinos para la salud o el medio ambiente. Recuperado de: http:// www.rap-al.org/articulos_files/La\%20Lista\%20de\%20Listas.pdf

RODRÍGUEZ, T., YOUGLOVE, L., LU, C., FUNEZ, A., WEPPNER, S., BARR, D., et al. (2006). Biological monitoring of pesticide exposures among applicators and their children in Nicaragua. Int J Occup Environ Health, 12(4), 312-320. 
ROHLMAN, D., ANGER, W. K. Y LEIN, P. J. (2011). Correlating neurobehavioral performance with biomarkers of organophosphorous pesticide exposure. Neurotoxicology, 32(2), 268-276.

ROSAS, L. Y ESKENAZI, B. (2008). Pesticides and child neurodevelopment. Curr Opin Pediatr, 20(2), 191-197.

ROTHLEIN, J., ROHLMAN, D., LASAREV, M., PHILLIPS, J., MUNIZ, J. Y MCCAULEY, L. (2006). Organophosphate pesticide exposure and neurobehavioral performance in agricultural and nonagricultural Hispanic workers. Environ Health Perspect, 114(5), 691-696.

THUNDIYIL, J., STOBER, J., BESBELLI, N., Y PRONCZUK, J. (2008). Acute pesticide poisoning: a proposed classification tool. Bulletin of the World Health Organization, 86(3). Recuperado de: http://www.who.int/bulletin/ volumes/86/3/07-041814.pdf

VAN DIJK, H., HOUGHTON, J., VAN KLEEF, E., VAN DER LANS, I., ROWE, G. Y FREWER, L. (2008). Consumer responses to communication about food risk management. Appetite, 50, 340-352.

VENTURA, C., NÚÑEZ, M., MIRET, N., MARTINEL LAMAS, D., RANDI, A., VENTURINO, A., RIVERA, E. Y COCCA, C. (2012). Differential mechanisms of action are involved in chlorpyrifos effects in estrogen-dependent or -independent breast cancer cells exposed to low or high concentrations of the pesticide. Toxicology Letters, 213, 184-193.

VIDA, P. Y MORETTO, A. (2007). Pesticide exposure pathways among children of agricultural workers. J Public Health, 15, 289-299.

WADDELL, B. L., ZAHM, S. H., BARIS, D., WEISENBURGER, D. D., HOLMES, F., BURMEISTER, L. F., et al. (2001). Agricultural use of organophosphate pesticides and the risk of non-Hodgkin's lymphoma among male farmers (United States). Cancer Causes Control, 12(6), 509-517. 